\title{
Responding on a multiple schedule of reinforcement as a function of different septal lesions in the rat
}

\author{
ALEX POPLAWSKY \\ Bloomsburg University of Pennsylvania, Bloomsburg, Pennsylvania
}

\begin{abstract}
The response rates of rats with either control operations or lateral, medial, or complete septal lesions were compared on a multiple fixed-interval (FI)/random-interval (RI) 40-sec schedule of reinforcement in which the rat was required to respond on a different lever for each alternating schedule component. Rats with lateral septal lesions responded at higher rates during the first 8-sec period of the FI component and had poorer response patterning than rats with control operations on the FI 40-sec reinforcement schedule. On the other hand, rats with medial septal lesions responded at higher rates than controls during the RI 40-sec component. Rats with complete septal lesions switched levers more than did controls before the ongoing schedule was completed. The results suggest that the lateral and medial septum have different functional roles mediating responding for food on interval schedules of reinforcement.
\end{abstract}

Rats with septal lesions generally respond at higher rates than controls under different schedules of reinforcement. For instance, rats with septal damage overresponded when their leverpressing behavior was controlled by a differential reinforcement of low rates of responding (DRL) schedule (Ellen, Wilson, \& Powell, 1964), a fixed-interval (FI) schedule (Beatty \& Schwartzbaum, 1968; Ellen \& Powell, 1962; Gay, 1977), and a variable-interval (VI) schedule of reinforcement (Dickinson, 1973; Poplawsky \& Hoffman, 1979).

Beatty and Schwartzbaum (1978) reported that septal rats had a higher percentage of responses in the first half of a FI 60 -sec schedule early in postoperative training; however, by Postoperative Session 14, the responding of the septal rats in the first half of the FI 60 was equivalent to controls. Under simple FI schedules of reinforcement, septal lesions generally resulted in an increased terminal response rate, and this was the major factor contributing to the increase in overall response rate (Beatty \& Schwartzbaum, 1968; Ellen \& Powell, 1962). Other FI studies (Dickinson, 1972; Poplawsky \& Cohen, 1977) have found that rats with septal lesions did not increase their response rates or show a disruption in the temporal patterning. In another study that used a second-order schedule of reinforcement, Cohen and Poplawsky (1979) found similar response rates for septal and control rats on a simple FI 60-sec schedule; however, when the rats were switched to a second-order schedule, rats with septal lesions re-

The author's mailing address is: Department of Psychology, Bloomsburg University of Pennsylvania, Bloomsburg, Pennsylvania 17815. sponded with higher response rates on the FI 60 -sec component. Their results suggest that, since the terminal rates of control and septal rats were very high after extended training ( 25 sessions), these high rates may have masked any changes produced by the septal lesion.

Studies using VI schedules of reinforcement found that septal lesions resulted in higher rates of responding than did control operations (Dickinson, 1973; Ellen, Gillenwater, \& Richardson, 1977; Poplawsky $\&$ Hoffman, 1979). This increase in response rate remained stable after extended postoperative training.

The purpose of the present experiment was to further investigate the effects of septal brain damage on responding during FI and random-interval (RI) schedules, using a multiple schedule of reinforcement in which a FI 40-sec schedule would be in effect on one lever while a RI 40 -sec schedule would be in effect on the other lever. Each schedule would alternate and would be signaled by a cue light above the appropriate lever. To insure stable behavior, testing consisted of 10 sessions that occurred following 25 sessions of baseline training. Besides measuring relative FI and RI performance, the two levers were used to assess any differences in the ability of rats with septal lesions to switch levers after reinforcement and continue to respond on the appropriate lever. Other studies (Donovick, 1968; Donovick, Burright, \& Swidler, 1973) showed that rats with septal damage have deficits in spatial alternation in a T-maze, while this study tested spatial alternation using an operant analog. Also, several studies have found differential effects on behavior as a function of destruction of the lateral or medial septal nuclei (Feldon \& Gray, 1979a, 1979b; Lancaster \& 
Johnson, 1976; Poplawsky \& Hoffman, 1979; Poplawsky \& Johnson, 1973). For instance, Feldon and Gray (1979a, 1979b) found that rats with medial septal lesions were more resistant to extinction in a straight alley than rats with lateral septal lesions after continuous or partial reinforcement. On the other hand, lateral septal lesions blocked the partial reinforcement effect, while medial septal lesions did not. When response suppression was caused by shock punishment, rats with lateral septal lesions responded at a higher rate than rats with medial septal lesions, while rats with medial septal lesions were able to suppress responding to the shock punishment (Feldon, Rawlins, \& Gray, 1982; Poplawsky \& Hoffman, 1979). Clearly, these different behavioral effects after selective septal lesions imply that the lateral and medial septal nuclei have different functions with respect to response suppression. Therefore, to provide more complete information to the understanding of FI, RI, and spatial alternation performance after septal damage, the effects of lateral, medial, and complete septal lesions on performance of a two-lever multiple FI-RI schedule of reinforcement were tested in the present experiment.

\section{METHOD}

\section{Animals}

Twenty-four adult male albino rats of the Sprague-Dawley strain (Camm Research, NJ), weighing between 370 and $500 \mathrm{~g}$ (100\% body weights) were used in this experiment. Each rat was individually housed with free access to water and maintained at $80 \%$ free-feeding body weights throughout the experiment. The colony room had controlled lighting with a 12-h light-dark cycle, with lights on at $0700 \mathrm{~h}$. Testing occurred between 0900 and $1700 \mathrm{~h}$.

\begin{abstract}
Apparatus
A two-lever operant chamber, $25.4 \mathrm{~cm}$ wide, $27.9 \mathrm{~cm}$ long, and $30.5 \mathrm{~cm}$ high (Coulbourn modular test cage, Model E10-10), was housed within an isolation cubicle. The levers were located on the right and left front panels $2.5 \mathrm{~cm}$ above the grid floor, while the cue lamps were located $4.5 \mathrm{~cm}$ above the levers. Food reinforcement consisted of a $45-\mathrm{mg}$ Noyes food pellet delivered by a pellet trough located in the center front panel $2.5 \mathrm{~cm}$ above the grid floor. A houselight located in the center of the front panel $23.5 \mathrm{~cm}$ above the grid floor illuminated the chamber when the session was in progress. Solid state modules controlled experimental sessions.
\end{abstract}

\section{Procedure}

Surgery and Histology. The rats were placed randomly into four equal groups $(n=6)$. They were anesthetized with ethyl ether and placed in a Kopf stereotaxic instrument. The appropriate anodal electrolytic lesions were accomplished stereotaxically by a stainless steel electrode insulated except for $0.5 \mathrm{~mm}$ at the tip. The lesion parameters for each group were as follows: complete septal lesions, $10.0 \mathrm{~mm}$ anterior to the frontal zero plane, $\pm 0.75 \mathrm{~mm}$ lateral to the midline, and $5.25 \mathrm{~mm}$ below the surface of the brain, with a current of $1.5 \mathrm{~mA}$ passed for $20 \mathrm{sec}$; for medial septal lesions, $10.0 \mathrm{~mm}$ anterior to the frontal zero plane, midline, and $5.5 \mathrm{~mm}$ below the surface of the brain, with a current of $1.5 \mathrm{~mA}$ passed for $13 \mathrm{sec}$; for lateral septal lesions, $10.0 \mathrm{~mm}$ anterior to the frontal zero plane, $\pm 0.75 \mathrm{~mm}$ lateral to the mid- line, $4.25 \mathrm{~mm}$ below the surface of the brain, with a current of $1.5 \mathrm{~mA}$ passed for $10 \mathrm{sec}$; and for control operations, $10.0 \mathrm{~mm}$ anterior to the frontal zero plane, $\pm 0.75 \mathrm{~mm}$ lateral to the midline, and $4 \mathrm{~mm}$ below the surface of the brain, with no current passed through the electrode. In all cases, the electrode was placed stereotaxically with the skull in a horizontal plane.

After behavioral (postoperative) observations were completed, all animals were anesthetized with an overdose of ethyl ether and perfused intracardially with $0.9 \%$ physiological saline solution followed by $10 \%$ formal-saline $(0.9 \% \mathrm{NaCL})$ solution. Brains were extracted, and frozen brain sections were made every $80 \mu$. To determine the location and extent of the lesion, every second section was mounted on a slide, stained with cresyl violet, and examined microscopically. An anterior, middle, and posterior coronal section of each animal's brain was projected on the appropriate Pellegrino and Cushman (1967) atlas-reference coronal planes, and reconstruction of the lesions were used to determine neural damage.

Training. Five days after surgery, when all rats returned to their free-feeding weights, the animals were placed on a restricted feeding regimen and maintained at $80 \%$ of their free-feeding body weights for the duration of the experiment. After magazine training, the rats were shaped to press the lever with the cue light on above it for 50 reinforcements for one session of continuous reinforcement (CRF). For the next five sessions, a response on the left lever in the presence of the left cue light produced a food pellet, extinguished the left cue light, and turned on the right cue light; a response on the right lever produced a food pellet, extinguished the right cue light, and turned on the left cue light. Therefore, both the cue lights and pellet delivery served as discriminative stimuli for switching to the other lever. The CRF schedule alternated between levers until 25 food pellets were obtained on each lever. A leverpress was reinforced only when the cue light above the lever was on; responses on the opposite lever were recorded as errors.

During the next five sessions, an FI 10-sec schedule and a random interval (RI) 10-sec alternated between levers. The first response on the left lever after $10 \mathrm{sec}$ from cue light onset produced a food pellet, extinguished the left cue light, and initiated the right cue light; then the first response on the right lever, after a random interval of time that averaged $10 \mathrm{sec}$, produced food, extinguished the right cue light, and initiated the left cue light. The next five sessions consisted of an FI 20 -sec schedule and a RI 20-sec schedule. Finally, for the next 35 sessions, the rats were placed on an FI 40 -sec schedule on one lever and a RI 40-sec schedule (range 2-80 sec) on the other lever, for a total of 16 reinforcements on each schedule of reinforcement. Half of the rats had an FI on the right lever, while the other half had an RI on the right lever throughout training. Three incorrect responses (leverpress on incorrect lever) produced a 5-sec blackout (houselight and cue lights extinguished); during this time, the levers were inactive and all timers were on hold.

Statistical analyses. A $4 \times 10$ mixed-design analysis of variance was used to analyze the data. The between factor was groups (complete septal, medial septal, lateral septal, and control), and the within factor was the last 10 sessions of the multiple FI 40RI 40-sec schedule of reinforcement. The dependent variables were the response rates per minute during the RI 40 , FI 40 , and five bins of 8-sec intervals of the FI 40-sec schedule. Other dependent variables were the error rate, the percentage of total time in blackout, and the index of curvature. The index of curvature is a quantitative measure of the degree of response patterning within FI schedules (Fry, Kelleher, \& Cook, 1960; Gollub, 1964). An index of 0 represents an equal response rate within each bin of the FI. Greater numbers indicate greater FI curvature, reaching a maximum of .8 when the FI is divided into fifths. For example, in an FI $40-\mathrm{sec}$ schedule, an index of .8 means that all the responses were emitted during the last $8 \mathrm{sec}$ of the FI. Tukey HSD tests were used to assess differences between means when a significant effect was found. 


\section{RESULTS}

\section{Histology}

In rats with complete septal lesions, both the lateral and medial septal nuclei were totally destroyed. The septal lesions extended from the genu of the corpus callosum to the fornix. Medial septal lesions were generally restricted entirely within the medial septum; however, two rats sustained minimal unilateral septal damage. Lateral septal lesions bilaterally removed the lateral septal nuclei, sparing only the most ventral components of these nuclei. Figure 1 presents a composite reconstruction of representative complete, medial, and lateral septal lesions.

\section{Behavior}

Table 1 presents the response rates per minute for the FI 40-sec and the RI 40-sec schedules of reinforcement, along with the error rates per minute for all of the groups. Although rats with lateral septal damage had a higher FI response rate than did controls, this difference of 18 responses/min was not significant $[F(3,20)=2.20]$. When each of the five 8-sec periods of the FI 40 were analyzed, the only significant difference between the groups was found during the first bin. Rats with lateral septal lesions consistently responded at higher rates than the other groups during the first $8 \mathrm{sec}$ of the FI 40-sec schedule $[F(3,20)=5.44, p<.01]$ (see Figure 2). Although not significant, they also had a higher average response rate than the other groups in the last four

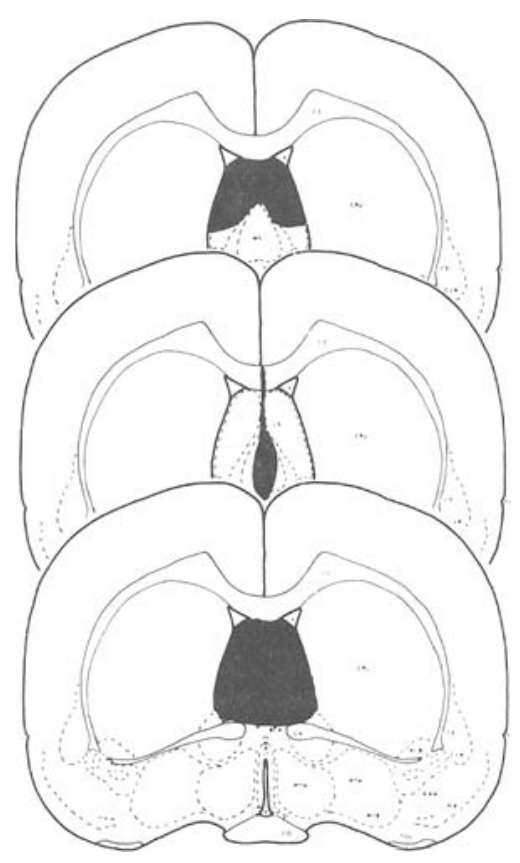

Figure 1. Reconstruction of the septal lesions at the point of maximum damage for lateral septal (top), medial septal (middle), and complete septal (bottom) lesions.
Table 1

Mean Responses Per Min for Each Group Over 10 Sessions

\begin{tabular}{lcccc} 
& \multicolumn{4}{c}{ Group } \\
\cline { 2 - 5 } Behavior & CS & LS & MS & C \\
\hline FI Rate & 18 & 43 & 33 & 25 \\
RI Rate & 26 & 29 & 48 & 19 \\
Error Rate & 8.5 & 5.9 & 3.9 & 5.2 \\
\hline
\end{tabular}

Note-CS = complete septal lesion; $L S=$ lateral septal lesion; $M S=$ medial septal lesion; $C=$ control.

8-sec periods. This, along with the significantly higher rate in the first bin, resulted in poor FI patterning. Figure 3 presents the index of curvature for each group over the last 10 sessions. Rats with lateral septal lesions had the poorest response patterning on the FI $\mathbf{4 0}$ with an index of curvature of .29 , whereas rats with control operations had an index of .46 over the 10 sessions $[F(3,20)=4.45$, $p<$ .025].

Figure 4 shows that when a RI 40 -sec schedule was in effect, rats with medial septal lesions consistently had a higher response rate than controls $[F(3,20)$ $=4.77, \mathrm{p}<.025$ ]. Differences in error rates (see Table 1) revealed that rats with complete septal lesions had a higher error rate than rats with medial septal lesions $[t(20)=2.56, p<.05]$; however, there were no significant differences between these two groups and controls. This high error rate for rats with complete septal lesions resulted in a higher percentage of time in the blackout condition $(25 \%)$ compared with controls $(14 \%)$ or rats with medial septal lesions $[10 \% ; F(3,20)=3.65, p<.05]$.

All the dependent variables were consistent over sessions with only one significant session's effect for error rate $[F(9,180)=4.61, p<.01]$. Consequently, there were no other significant sessions effects or significant groups $\times$ sessions interactions.

\section{DISCUSSION}

The results of this study showed that there was a differential effect of lateral and medial septal lesions on the performance of a multiple FI-RI schedule of reinforcement. Specifically, rats with lateral septal lesions resulted in higher rates of responding during the first 8-sec period of the FI 40 along with poorer response patterning than controls, while rats with medial septal lesions had higher response rates than controls on the RI 40-sec schedule of reinforcement. Rats with complete septal lesions had difficulty alternating levers at the termination of each componentreinforcement schedule, and had an increased tendency to switch levers within components. This behavior resulted in the highest error rate and also the highest percentage of blackout time. 


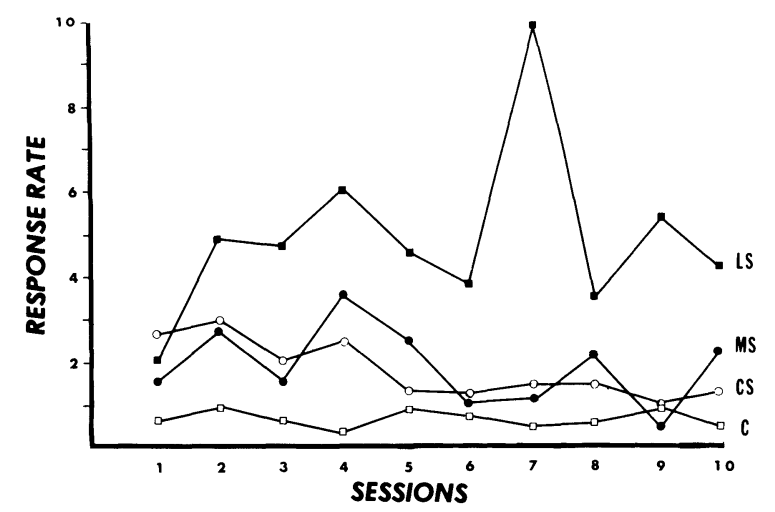

Figure 2. Mean response rate per minute for each group during the first 8-sec period of the FI 40 -sec reinforcement schedule over 10 sessions. Abbreviations: $\mathbf{L S}=$ lateral septal lesion, $\mathrm{MS}=$ medial septal lesion, $\mathbf{C S}=$ complete septal lesion, $\mathrm{C}=$ control operation.

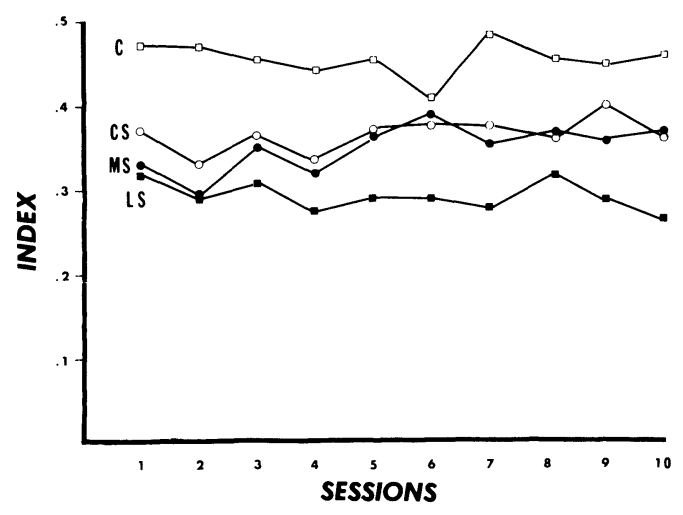

Figure 3. Mean index of curvature for each group over 10 sessions. Abbreviations: $\mathbf{L S}=$ lateral septal lesion, $\mathrm{MS}=$ medial septal lesion, $\mathrm{CS}=$ complete septal lesion, $\mathrm{C}=$ control operation.

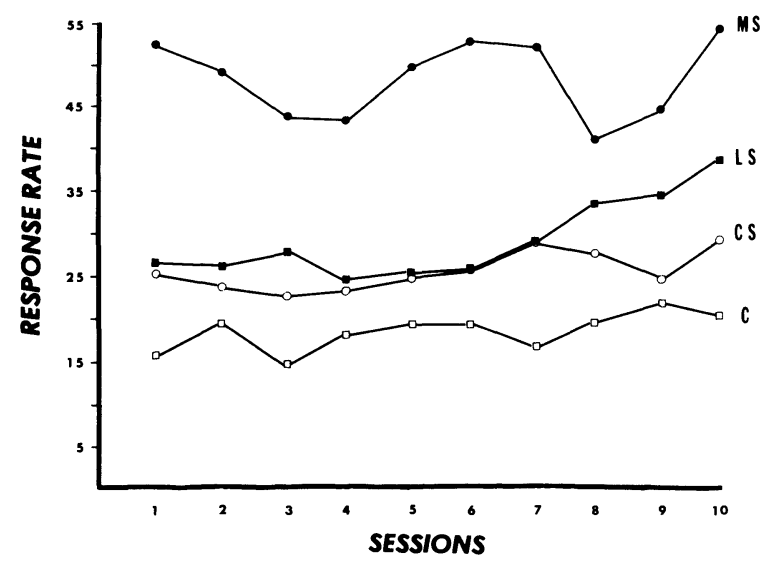

Figure 4. Mean response rate per minute for each group during the RI 40-sec reinforcement schedule over 10 sessions. Abbreviations: $\mathbf{L S}=$ lateral septal lesion, $\mathrm{MS}=$ medial septal lesion, $\mathrm{CS}=$ complete septal lesion, $\mathrm{C}=$ control operation.
Beatty and Schwartzbaum (1968) found that rats with complete septal lesions had a temporary increase in response rate early in an FI 60 -sec reinforcement schedule that returned to control levels by Session 14 . In the present study, rats with lateral septal lesions had a long-lasting increase in response rate in the first 8-sec period of the FI $\mathbf{4 0}$ that was still present 80 days after surgery and after 50 sessions of operant experience. Lateral septal damage also resulted in a consistently low index of curvature, which indicates a poor response pattern over the 40 -sec time interval preceding reinforcement. During the FI component used in the present study, the rats could have delayed reinforcement and extended the FI by responding on the incorrect lever, producing a blackout period for $5 \mathrm{sec}$. This contingency makes it difficult to directly compare the results of studies that used a simple FI (Beatty \& Schwartzbaum, 1968; Gay, 1977) with the present multiple FI-RI reinforcement schedule. However, it appears that another factor, besides FI training, that influences responding early in the FI is the location of the septal lesions, that is, rats with lateral septal lesions overrespond during early segments of an FI reinforcement schedule.

A number of studies have found that, on a simple VI, the response rates of rats with complete septal lesions were higher than those of controls (Atnip \& Hothersall, 1975; Dickinson, 1973; Poplawsky \& Hoffman, 1979). In the present multiple FI-RI study, rats with medial septal lesions had higher response rates than did controls on the RI 40-sec component. This agrees with the findings of Feldon, Rawlins, and Gray (1982), who also found that medial septal lesions resulted in higher response rates on a RI 64sec reinforcement schedule. In comparing the components of the multiple schedule of reinforcement, it was evident that rats with lateral or medial septal lesions responded differently when the FI or RI schedules of reinforcement were in effect. For instance, rats with lateral septal lesions decreased their response rates approximately at the same proportion as did controls on the RI relative to the FI, whereas rats with medial septal lesions, as well as rats with complete septal lesions, increased their response rates on the RI relative to their overall FI rates. Rats with medial septal lesions overresponded to the unpredictability of reinforcement on the RI schedule, and did not suppress responding as readily as rats with lateral septal lesions during the RI 40-sec component. This explanation agrees with Feldon et al.'s (1982) hypothesis that medial septal lesions weaken nonreward-induced suppression, and, therefore, these rats respond more often under conditions of nonreward.

Rats with complete septal lesions had difficulty in alternating levers after reward as well as in continuing to respond on the correct lever. This behav- 
ioral deficit makes it difficult to compare the response rates of rats with complete septal damage with the response rates of other groups on the different reinforcement schedules. Donovick et al. (1973) also found that rats with septal lesions had great difficulty on a simple spatial alternation task in a T-maze when tested 7 weeks after surgery. In the present study, rats with complete septal lesions had more errors than controls, and had the most blackout time. On the other hand, rats with medial septal lesions had significantly lower error rates than complete septals, and were able to switch levers as efficiently as controls. This suggests that rats with complete septal damage have difficulty with spatial alternation, that is, switching from a response that was previously rewarded to another similar response requiring a different spatial orientation.

The results of the present study indicate that lesions of the medial, lateral, or complete septum result in changes in behavior that are associated with different interval-reinforcement conditions. This agrees with several studies that suggest that the medial and lateral septum may act in opposition to each other in a variety of behavioral settings. For instance, rats with medial septal lesions have larger social contact times (Poplawsky \& Johnson, 1973), show more shock-induced suppression of a barpress response (Feldon et al., 1982; Poplawsky \& Hoffman, 1979), and take longer to extinguish a straight alley running behavior (Feldon \& Gray, 1979a, 1979b) than rats with lateral septal lesions. Changes in response rates and other behaviors not only are a function of the anatomical loci of the septal damage, but also may change as a function of postoperative training. There is a need for further research to specify the time course of behavioral changes that occur in operanttest situations following septal brain damage.

\section{REFERENCES}

Atnip, G., \& Hothersall, D. Response suppression in normal and septal rats. Physiology \& Behavior, 1975, 15, 417-421.

Beatty, W. W., \& Schwartzbaum, J. S. Commonality and specificity of behavioral dysfunctions following septal and hippocampal lesions in rats. Journal of Comparative and Physiological Psychology, 1968, 66, 60-68.

Cohen, S. L., \& Poplawsky, A. The effects of septal lesions on second-order schedules. Behavioral and Neural Biology, 1979, 27, 175-186.

Dickinson, A. Septal damage and response output under frustra- tive nonreward. In R. A. Boakes \& M. S. Halliday (Eds.), Inhibition and learning. London: Academic Press, 1972.

Dickinson, A. Septal lesions in rats and the acquisition of freeoperant successive discriminations. Physiology \& Behavior, $1973,10,305-313$.

Donovick, P. J. Effects of localized septal lesions on hippocampal EEG activity and behavior in rats. Journal of Comparative and Physiological Psychology, 1968, 66, 569-578.

Donovick, P. J., Burright, R. G., \& Swidler, M. A. Presurgical rearing environment alters exploration, fluid consumption, and learning of septal lesioned and control rats. Physiology \& Behavior, 1973, 11, 543-553.

Ellen, P., Gillenwater, G., \& Richardson, W. K. Extinction responding by septal and normal rats following acquisition under four schedules of reinforcement. Physiology \& Behavior, 1977, 18, 609-615.

Ellen, P., \& Powell, E. W. Effects of septal lesions on behavior generated by positive reinforcement. Experimental Neurology, 1962, 6, 1-11.

Ellen, P., Wilson, S., \& Powell, E. W. Septal inhibition and timing behavior in the rat. Experimental Neurology, 1964, 10, 120-132.

Feldon, J., \& Gray, J. A. Effects of medial and lateral septal lesions on the partial reinforcement extinction effect at one trial a day. Quarterly Journal of Experimental Psychology, 1979, 31, 653-674. (a)

Feldon, J., \& Gray, J. A. Effects of medial and lateral septal lesions on the partial reinforcement extinction effect at short inter-trial intervals. Quarterly Journal of Experimental Psychology, 1979, 31, 675-690. (b)

Feldon, J., Rawlins, J.N.P., \& Gray, J. A. Effects of lateral and medial septal lesions on response suppression maintained by response-contingent and response-independent shock. Physiological Psychology, 1982, 10, 145-152.

Fry, W., Kelleher, R. T., \& Cook, L. A. A mathematical index of performance on fixed-interval schedules of reinforcement. Journal of the Experimental Analysis of Behavior, 1960, 3, 193-199.

GAY, P. E. Activity levels and fixed interval performance in rats with septal lesions. Behavioral Biology, 1977, 20, 534-540.

GolluB, L. R. The relations among measures of performance on fixed-interval schedules. Journal of the Experimental Analysis of Behavior, 1964, 7, 337-343.

LANCASter, J., \& Johnson, D. A. Effects of anterior, posterior, medial, lateral, and total septal lesions upon fixed ratio performance in the rat. Physiology \& Behavior, 1976, 17, 1-7.

Pellegrino, L. J., \& Cushman, A. J. A stereotaxic atlas of the rat brain. New York: Appleton-Century-Crofts, 1967.

Poplawsky, A., \& Cohen, S. L. Septal lesions and the reinforceromission effect. Physiology \& Behavior, 1977, 18, 983-985.

Poplawsky, A., \& Hoffman, S. L. Effects of lateral, medial, or complete septal lesions on response suppression. Physiology \& Behavior, 1979, 22, 679-682.

Poplawsky, A., \& Johnson, D. A. Open-field social behavior of rats following lateral or medial septal lesions. Physiology \& Behavior, 1973, 11, 845-854.

(Manuscript received February 11, 1983; revision accepted for publication April 11, 1983.) 